\title{
Bariatric surgery and non-alcoholic fatty liver disease: current and potential future treatments
}

\author{
Akira Sasaki *, Hiroyuki Nitta, Koki Otsuka, Akira Umemura, Shigeaki Baba, Toru Obuchi and \\ Go Wakabayashi
}

Department of Surgery, Iwate Medical University School of Medicine, Morioka, Japan

Edited by:

María M. Malagón, University of

Cordoba, Spain

Reviewed by:

Bruno Bonaz, Grenoble Faculty of Medicine and Hospital, France Javier Gomez-Ambrosi, Clinica

Universidad de Navarra, Spain

\section{*Correspondence.}

Akira Sasaki, Department of Surgery,

Iwate Medical University School of

Medicine, Uchimaru 19-1, Iwate,

Morioka 020-8505, Japan

e-mail:sakira@iwate-med.ac.jp
Non-alcoholic fatty liver disease (NAFLD) and non-alcoholic steatohepatitis (NASH) are increasingly common cause of chronic liver disease worldwide. The diagnosis of NASH is challenging as most affected patients are symptom-free and the role of routine screening is not clearly established. Most patients with severe obesity who undergo bariatric surgery have NAFLD, which is associated insulin resistance, type 2 diabetes mellitus (T2DM), hypertension, and obesity-related dyslipidemia. The effective treatment for NAFLD is weight reduction through lifestyle modifications, antiobesity medication, or bariatric surgery. Among these treatments, bariatric surgery is the most reliable method for achieving substantial, sustained weight loss. This procedure is safe when performed by a skilled surgeon, and the benefits include reduced weight, improved quality of life, decreased obesity-related comorbidities, and increased life expectancy. Further research is urgently needed to determine the best use of bariatric surgery with NAFLD patients at high risk of developing liver cirrhosis and its role in modulating complications of NAFLD, such as T2DM and cardiovascular disease. The current evidence suggests that bariatric surgery for patients with severe obesity decreases the grade of steatosis, hepatic inflammation, and fibrosis. However, further long-term studies are required to confirm the true effects before recommending bariatric surgery as a potential treatment for NASH.

Keywords: non-alcoholic fatty liver disease, non-alcoholic steatohepatitis, obesity, diabetes, bariatric surgery, laparoscopy

\section{INTRODUCTION}

In recent years, the obese population has been rapidly increasing because of increases in diets rich in saturated fat and processed carbohydrates and sedentary lifestyles. The metabolic syndromes that are considered risk factors of arteriosclerotic diseases, such as abnormal glucose tolerance, hyperlipemia, and hypertension, have increased alongside obesity in the developed world (1). Non-alcoholic fatty liver disease (NAFLD) is a liver phenotype of metabolic syndrome. Factors that affect the morbidity of this disease include genetic background, an epigenetic control mechanism, the fat toxicity of free fatty acid, and a natural immunity system of intestinal bacteria. Based on current trends, the rate of obesity in the US is projected to reach approximately $40 \%$ by 2025 (2). However, the prevalence of NAFLD is not well known, though it is estimated at $6.3-33 \%$ worldwide. In North America, the prevalence of NAFLD is estimated at about $20 \%$, and the prevalence of its more progressive subtype, non-alcoholic steatohepatitis (NASH), is approximately $2-3 \%(3-6)$.

Non-alcoholic fatty liver disease is the most common cause of liver dysfunction worldwide, and obesity is a well-documented risk factor for the disease. It is highest in populations with preexisting metabolic conditions, such as obesity, T2DM, and dyslipidemia (7). NASH is often an incidental diagnosis and can be associated with other disease processes, such as hypopituitarism, hypogonadism, hypothyroidism, polycystic ovarian syndrome, and obstructive sleep apnea syndrome. It is also frequently associated with pancreatoduodenal resections (8).

Perioperative liver biopsy in patients with severe obesity can result in complications, such as bleeding and sampling error, making exact diagnosis difficult to achieve. Among patients with severe obesity undergoing bariatric surgery, the prevalence of NAFLD can exceed $90 \%$, and up to $5 \%$ of these patients may have unsuspected cirrhosis. Bariatric surgery can improve obesity-related diseases in addition to reducing body weight; as a result, it has more recently been termed "metabolic surgery."

We conducted a literature review using the Medline database. We identified studies conducted between 1995 and 2014 that examined the impact of bariatric surgery on NAFLD. This review evaluates the benefits and potential treatment option of bariatric surgical procedures for NAFLD as described in the literature.

\section{MEDICAL TREATMENT OF NAFLD}

Past studies investigating lifestyle changes, such as diet, exercise, and behavior modification that resulted in weight loss of $5-10 \%$, have shown that these changes can improve steatosis in some patients $(7,9)$. Greater weight loss of at least $10 \%$ has also been shown to improve inflammation. However, it is important to note that adherence to lifestyle interventions can be problematic. In several studies, only $15 \%$ of patients achieved a weight loss $>10 \%$, and most of these people regained the weight. The best average 
weight loss among participants has been reported at $3-4 \mathrm{~kg}$ at 2 and 4 years (10).

Although no drugs are specifically licensed for the treatment of NAFLD, there is evidence to support the use of selected agents. Antiobesity medications such as orlistat, and drugs that augment insulin sensitivity and reduce plasma glucose concentrations and oxidative stress such as thiazolidinediones and metformin are among those that have been shown to improve liver histology in NAFLD (11-14). Orlistat inhibits enteric lipid absorption and has been promoted as a weight loss aid. It has also been evaluated as a potential therapy for NASH (15).

It is thought that GLP-1 receptor agonists work toward improvement of insulin resistance because not only can they improve the effect of insulin secretion but can also promote glucose uptake by the liver, adipose tissue, and skeletal muscle (16). Many studies have reported improvement outcomes with GLP-1 receptor agonist therapy in animal NAFLD models and NAFLD patients, and the expectation of this drug's effectiveness to treat NAFLD is increasing (17-19). According to a recent systematic review and meta-analysis, GLP-1 receptor agonists significantly decreased the weight of obese patients regardless of the existence of T2DM (20). Astrup et al. planned a clinical study of the GLP-1 receptor agonists to treat severely obese patients without T2DM. These researchers reported that weight loss was significantly greater in the patients treated with GLP-1 receptor agonists than in the patients with only diet and exercise modifications. These results showed better outcomes than in the patients with orlistat as a comparative drug (21). Thus, the use of pharmacological agents for obesity is quickly gaining popularity for patients with mild obesity and visceral adipose obesity. Incretin mimetics may therefore represent a novel therapeutic option in the future for slowing the progression of NAFLD. However, larger studies with longer treatment durations are required to confirm whether incretin mimetics confers any benefits above their effects on weight loss.

\section{BARIATRIC SURGERY AND NAFLD}

The goal of bariatric surgery is not only to achieve satisfactory weight loss but also to obtain improvements in obesity-related comorbidities, including T2DM, obstructive sleep apnea syndrome, hyperlipidemia, and hypertension. However, no randomized controlled trials examining the effects of bariatric surgery on NAFLD were found in the literature search. Two meta-analyses evaluated the effect of bariatric surgery on the liver histology of patients with NAFLD $(22,23)$. Mummadi et al. reported that the improvement or resolution rates of steatosis, steatohepatitis, and fibrosis of 15 studies and 766 paired liver biopsies after bariatric surgery were $91.6,81.3$, and $65.5 \%$, respectively (22). Complete resolution of NASH was archived in $69.5 \%$ of patients. However, a recently published Cochrane review concluded that the lack of randomized clinical trials or quasi-randomized clinical studies has prevented a definitive assessment of the benefits and harms of bariatric surgery as a therapeutic approach for patients with NASH (23).

\section{ROUX-EN-Y GASTRIC BYPASS}

Bariatric surgery has a positive effect on obesity-related diseases in addition to reducing body weight. Because of this, it has been more recently termed metabolic surgery. Roux-en-Y gastric bypass (RYGB) has a reportedly greater positive effect on T2DM than restrictive procedures. This mechanism is not understood; however, two theories exist: the hindgut and foregut hypotheses. In the hindgut hypothesis, bypass of the upper small intestine diverts food directly to the lower small intestine, where glucagon-like peptide-1 (GLP-1) secretory cells promote GLP-1 secretion. In the foregut hypothesis, an unknown glucose-tolerance-complicating factor that inhibits GLP-1 secretion is discharged from the upper small intestine; because bypass surgery prevents food from passing through the upper small intestine, this factor is no longer secreted $(24,25)$. Post-operatively, patients who undergo RYGB have shown a marked reduction in hepatic lipid content and improved hepatic insulin sensitivity well before significant weight loss occurs. These benefits to the liver are directly related to at least two enteroendocrine cells-synthesized gut peptides GLP-1 and peptide YY (26-29).

No randomized clinical trials of bariatric surgery in NAFLD were found in the literature. Fourteen studies including reviews showed that RYGB has been associated with improvement in NAFLD (11, 28, 30-42) (Table 1). Overall, RYGB in obese patients has decreased the grade of steatosis, hepatic inflammation, and fibrosis. However, some studies reported a few patients with worsening or new fibrosis $(30,32,33,35,37)$.

\section{SLEEVE GASTRECTOMY AND GASTRIC BANDING}

Recent studies have shown that laparoscopic sleeve gastrectomy (LSG) is safe and effective, resulting in weight loss somewhere between the rates of laparoscopic RYGB and laparoscopic adjustable gastric banding (LAGB) $(43,44)$. Although LSG is a restrictive procedure, the removal of the gastric fundus, the primary site of ghrelin production, appears to have a hormonal effect that enhances weight loss by reducing appetite (25).

Based on questionnaire survey results from 50 countries, Buchwald et al. reported that 340,768 bariatric surgeries were performed worldwide in 2011 (45), which included 47\% LRYGB, 28\% LSG, and 18\% LAGB. LSG, an initial bariatric surgery for severely obese patients, is a technique used to lower the rates of complication and surgical death. For these reasons, LSG has increased rapidly worldwide and is predicted to become the most frequently performed bariatric surgery.

Only four studies were found that utilized LAGB or LSG as a restrictive procedure to examine the effects on NAFLD (4649) (Table 2). Dixon et al. conducted paired liver biopsies on 36 obese patients - the first at the time of LAGB and the second after weight loss. On follow-up biopsies at 25.6 months after LAGB, the mean percentage of excess weight loss was $52 \%$, and there were major improvements in lobular steatosis, necroinflammatory changes, and fibrosis. However, portal abnormalities remained unchanged (46). The same author examined a second similar investigation of 60 severely obese patients after LAGB. On follow-up biopsies at 29.5 months after LAGB, only $10 \%$ displayed NASH. Improvements in steatosis, inflammation, and fibrosis, as well as in biochemical markers of liver function, were seen in all of the patients. The researchers concluded that decreases in gamma-glutamyl transferase concentrations were associated with histological improvement (47). Mathurin et al. reported no significant differences among LAGB, RYGB, and biliointestinal bypass 
Table 1 | Effect of RYGB on NAFLD

\begin{tabular}{|c|c|c|c|c|}
\hline Reference & Patients & Types of study & Main outcomes (improvement) & Follow-up (months) \\
\hline Silverman et al. (30) & 91 & Retro & Steatosis and fibrosis & 18.4 \\
\hline Mattar et al. (31) & 70 & Pros & Steatosis and fibrosis & 15 \\
\hline Clark et al. (32) & 16 & Pros & Steatosis, inflammation, and fibrosis & 0.8 \\
\hline Mottin et al. (33) & 90 & Retro & Steatosis $(82 \%)$ & 12 \\
\hline Klein et al. (34) & 7 & Pros & Fibrosis and inflammation & 12 \\
\hline Barker et al. (28) & 19 & Pros & Steatosis, inflammation, and fibrosis & 21.4 \\
\hline Csendes et al. (35) & 16 & Pros & Histology $(80 \%)$ & 22 \\
\hline de Almeida et al. (36) & 16 & Pros & Steatosis, inflammation, and fibrosis & 23.5 \\
\hline Furuya et al. (37) & 18 & Pros & Steatosis and fibrosis & 24 \\
\hline Liu et al. (38) & 39 & Retro & Steatosis, inflammation, and fibrosis & 18 \\
\hline Weiner et al. (39) & 116 & Retro & Complete regression (83\%) & 18.6 \\
\hline Meretto et al. (40) & 78 & Retro & Resolved fibrosis (50\%) & Unavailable \\
\hline Vargas et al. (41) & 26 & Pros & Steatosis, inflammation, and fibrosis & 16 \\
\hline Tai et al. (42) & 21 & Pros & Steatosis, inflammation, and fibrosis & 12 \\
\hline
\end{tabular}

Pros, prospective; Retro, retrospective.

Table 2 | Effect of restrictive bariatric surgery on NAFLD.

\begin{tabular}{|c|c|c|c|c|}
\hline Reference & Patients & Types of surgery & Main outcomes (improvement) & Follow-up (months) \\
\hline Dixon et al. (46) & 36 & LAGB & Steatosis, inflammation, and fibrosis & 25.6 \\
\hline Dixon et al. (47) & 60 & LAGB & Steatosis, inflammation, and fibrosis & 29.5 \\
\hline Mathurin et al. (48) & 381 & LAGB & Steatosis & 50 \\
\hline Karcz et al. (49) & 236 & LSG & AST, ALT, triglyceride and HDL levels & 12 \\
\hline
\end{tabular}

Pros, prospective; Retro, retrospective.

groups, and those who progressed to fibrosis became more insulin resistant. Five years after bariatric surgery for severe obesity, almost all of the patients had low levels of NAFLD, whereas fibrosis slightly increased (48).

Studies of follow-up biopsies for NAFLD in LSG were not found in the literature review. Karcz et al. reported on the effect on NASH diagnosed at the time of LSG and NASH-related comorbidities using clinical and biological data at 1- and 3-year follow-ups. A significant improvement of AST, ALT, triglyceride, and HDL levels was shown in the $87 \mathrm{NASH}$ patients (49).

As a mechanism that improves NAFLD, GLP-1 plays an important role after LSG. It has been shown that GLP-1 receptor agonists may be an effective tool for the treatment of NASH. This is due to the continuous increase of serum GLP-1 levels and the reduction of hepatic glucose production, which are expected indirect actions of GLP-1 after LSG. However, a future study is required to confirm this. Umemura et al. reported that the mean weight reduction was $11 \mathrm{~kg}$ at 1 month after LSG for patients with severe obesity; the mean GLP-1 levels increased, while mean ghrelin levels decreased significantly (50). The researchers also reported that the changes in these hormones might have derived from a resection of the gastric fundus and the hindgut effect with accelerated gastric emptying.

\section{BILIOPANCREATIC DIVERSION PROCEDURE}

Only two studies were found the biliopancreatic diversion procedures to evaluate the effects on NAFLD $(51,52)$. Keshishian et al. studied repeat liver biopsies on 78 patients after a duodenal switch procedure. Hepatic inflammation slightly worsened at 6 months, but improvements were seen at and beyond 12 months. By 36 months, the histological degree of steatosis had improved by $60 \%$ and the severity of inflammation improved by three grades (51). Kral et al. studied liver biopsies on 104 patients after a biliopancreatic diversion procedure. Steatosis grades decreased in correlation with weight loss as expected, but the researcher observed a post-operative increase in fibrosis in $40 \%$ of the patients, a decrease in $27 \%$, and no change in $33 \%$ (52). The researcher suggested that several factors such as alcohol, bacterial products, iron storage, deficiencies of antioxidants, protein, lipotropic factors, or the generation of reactive oxygen species might explain the development or lack of improvement in a few patients with severe fibrosis or cirrhosis.

\section{BARIATRIC SURGERY AS A POTENTIAL TREATMENT}

\section{FOR NAFLD}

The current hypothesis for the development of NAFLD is that obesity and insulin resistance increase the release of free fatty acids from adipocytes (53), and hepatic insulin resistance and hepatic steatosis precede the development of T2DM (54).

Bile acids are important regulators of energy balance through the unclear receptor farmesoid $\mathrm{X}$ receptor (FXR) and the Gprotein-coupled membrane receptor TGR5. Activation of FXR by bile acids after a meal induces synthesis of the intestinal peptide 


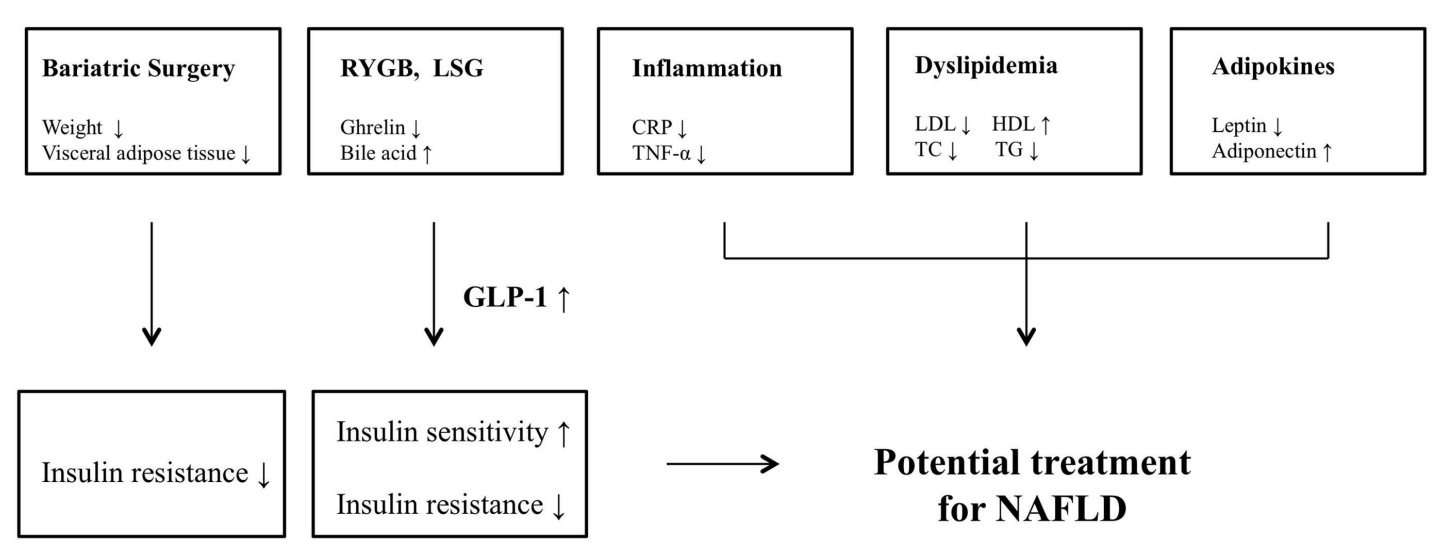

FIGURE 1 | Potential mechanisms or improvement for NAFLD after bariatric surgery.

hormone FGF-19 and triggers a cascade that controls fed-fasted state metabolism. Bile acids could also promote weight loss by increasing energy expenditure in brown adipose tissue via TGR5. Bile acids might be involved in bariatric surgery-induced changes in energy homeostasis through two mechanisms: increased secretion of bile acids with direct effects on energy balance and increased delivery to the distal intestine due to nutrient and bile rerouting, with increased stimulation of L-cell production and the release of hormones with attenuating effects on bariatric surgery (55). Patti et al. reported that total serum bile acid concentrations were higher in RYGB patients than in obese patients (56). Haluzíková et al. reported that the effects of LSG on serum concentrations of FGF-19 and FGF-21 along with circulating bile acids and other relevant hormonal and biochemical parameters (57). The researchers concluded that increased FGF-19 and decreased ghrelin concentrations could have partially contributed to the improvement of systemic inflammation and some metabolic parameters after LSG, while changes of FGF-21 are rather secondary because of weight loss. Pournaras et al. reported that gastric bypass leads to increased plasma bile acids, FGF-19, incretin, and satiety gut hormone concentrations (58).

In a recent systematic review, post-operative resolution or improvement of T2DM occurred in $73 \%$ of patients (59). Potential mechanisms of T2DM remission underlying the direct antidiabetic impact of bariatric surgery include enhanced nutrient stimulation of GLP-1, altered physiology from excluding ingested nutrients from the upper intestine, compromised ghrelin secretion, improved hepatic insulin sensitivity, and improved peripheral insulin sensitivity. The changes in the rate of eating, gastric emptying, intestinal transit time, nutrient absorption, and sensing, as well as bile acid metabolism, may also be implicated $(60,61)$. Bariatric surgery, which offers the effects of metabolic surgery, should be considered for T2DM patients having difficulty continuing with medical treatment and a potential for future deterioration and diabetic complications.

The mechanism of how bariatric surgery plays a role as a potential treatment for NAFLD is also complex and not fully understood. Bariatric surgery is likely to have potential benefits in ameliorating the factors such as insulin resistance, lipid profile, inflammation, weight loss, and adipokines that contribute in a marked way to the pathogenesis of NAFLD (Figure 1).

\section{CURRENT GUIDELINES}

Currently, the only proven effective treatment for NAFLD is weight loss. Many reports have shown that when performed by a skilled surgeon, bariatric surgery is safe and effective for reducing weight, improving quality of life, decreasing obesity-related diseases, and increasing life expectancy. However, there is a lack of randomized controlled trials examining the effects of bariatric surgery on NAFLD; the only available studies are either retrospective or prospective cohort studies (5).

The American Association for the Study of Liver Diseases (AASLD), American College of Gastroenterology (AGG), and American Gastroenterological Association (AGA) recommend that foregut bariatric surgery should not be contraindicated in otherwise eligible obese individuals with NAFLD or NASH. However, these organizations recognize that it is premature to consider foregut bariatric surgery as an established option to treat NASH (5).

\section{CONCLUSION}

The current evidence suggests that bariatric surgery for patients with severe obesity decreases the grade of steatosis, hepatic inflammation, and fibrosis. However, the lack of randomized clinical trials demonstrating the beneficial effects of bariatric surgery procedures for treatment of NAFLD prevented us from reaching a scientifically sustained conclusion. Positive results have been observed in cohort studies; however, this research has a high risk of bias. The research also reported a potential risk for worsening fibrosis scores. Therefore, bariatric surgery must be assessed in randomized clinical trials.

The mechanism of bariatric surgery's role as a potential treatment for NASH is complex and not fully understood. Further long-term studies are required to confirm the true effects before recommending bariatric surgery as a treatment option for NASH. 


\section{REFERENCES}

1. Patel AA, Torres DM, Harrison SA. Effect of weight loss on nonalcoholic fatty liver disease. J Clin Gastroenterol (2009) 43(10):970-4. doi:10.1097/MCG. 0b013e3181b57475

2. Kopelman PG. Obesity as a medical problem. Nature (2000) 404(6778):635-43. doi:10.1038/35007508

3. Younossi ZM, Reyes MJ, Mishra A, Mehta R, Henry L. Systematic review with meta-analysis: non-alcoholic steatohepatitis - a case for personalized treatment based on pathogenic targets. Aliment Pharmacol Ther (2014) 39(1):3-14. doi:10.1111/apt.12543

4. Paredes AH, Torres DM, Harrison SA. Nonalcoholic fatty liver disease. Clin Liver Dis (2012) 16(2):397-419. doi:10.1016/j.cld.2012.03.005

5. Chalasani N, Younossi Z, Lavine JE, Diehl AM, Brunt EM, Cusi K, et al. The diagnosis and managements of non-alcoholic fatty liver disease: practice Guideline by the American Association for the Study of Liver Diseases, American College of Gastroenterology, and the American Gastroenterological Association. Hepatology (2012) 55(6):2005-23. doi:10.1002/hep.25762

6. Karlas T, Wiegand J, Berg T. Gastrointestinal complications of obesity: nonalcholic fatty liver disease (NAFLD) and its sequelae. Best Pract Res Clin Endocrinol Metab (2013) 27(2):195-208. doi:10.1016/j.beem.2013.02.002

7. Attar BM, Van Thiel DH. Current concepts and management approaches in nonalcoholic fatty liver disease. ScientificWorldJournal (2013) 2013:481893. doi:10.1155/2013/481893

8. Tanaka N, Horiuchi A, Yokoyama T, Kaneko G, Horigome N, Yamaura T, et al. Clinical characteristics of de novo nonalcoholic fatty liver disease following pancreaticoduodenectomy. J Gastroenterol (2011) 46(6):758-68. doi:10.1007/ s00535-011-0370-5

9. Marra F, Lotersztajin S. Pathophysiology of NASH: perspectives for a targeted treatment. Curr Pharm Des (2013) 19(29):5250-69. doi:10.2174/ 13816128113199990344

10. Franz MJ, VanWormer JJ, Craiin AL, Boucher JL, Histon T, Caplan W, et al. Weight-loss outcomes: a systematic review and meta-analysis of weight-loss clinical trials with a minimum 1-year follow-up. J Am Diet Assoc (2007) 107(10):1755-67. doi:10.1016/j.jada.2007.07.017

11. Hafeez S, Ahmed MH. Bariatric surgery as potential treatment for nonalcoholic fatty liver disease: a future treatment by choice or by chance? J Obes (2013) 2013:839275. doi:10.1155/2013/839275

12. Lingvay I, Raskin P, Szczepaniak LS. Effect of insulin-metformin combination on hepatic steatosis in patients with type 2 diabetes. J Diabetes Complications (2007) 21(3):137-42. doi:10.1016/j.jdiacomp.2007.02.005

13. Bellentani S, Scaglioni F, Marino M, Bedogni G. Epidemiology of non-alcoholic fatty liver disease. Dig Dis (2010) 28(1):155-61. doi:10.1159/000282080

14. Hussein O, Grosovski M, Schlesinger S, Szvalb S, Assy N. Orlostat reverse fatty infiltration and improves hepatic fibrosis in obese patients with nonalcoholic steatohepatitis (NASH). Dig Dis Sci (2007) 52(10):2512-9. doi:10.1007/s10620006-9631- 1

15. Harrison SA, Fecht W, Brunt EM, Neuschwander-Tetri BA. Orlistat for overweight subjects with nonalcoholic steatohepatitis: a randomized, prospective trial. Hepatology (2009) 49(1):80-6. doi:10.1002/hep.22575

16. Drucker DJ. The biology of incretin hormones. Cell Metab (2006) 3(3):153-65. doi:10.1016/j.cmet.2006.01.004

17. Ding X, Saxena NK, Lin S, Gupta NA, Anania FA. Exendin-4, a glucagon-like protein-1 (GLP-1) receptor agonist, reverses hepatic steatosis in ob/ob mice. Hepatology (2006) 43(1):173-81. doi:10.1002/hep.21006

18. Trevaskis JL, Griffin PS, Wittmer C, Neuschwander-Tetri BA, Brunt EM, Dolman CS, et al. Glucagon-like protein-1 receptor agonism improves metabolic, biochemical, and histopathological indices of nonalcoholic steatohepatitis in mice. Am J Physiol Gastrointest Liver Physiol (2012) 302(8):G762-72. doi:10.1152/ajpgi.00476.2011

19. Kim YO, Schuppan D. When GLP-1 hits the liver: a novel approach for insulin resistance and NASH. Am J Physiol Gastrointest Liver Physiol (2012) 302(8):G759-61. doi:10.1152/ajpgi.00078.2012

20. Visbøll T, Christensen M, Junker AE, Knop FK, Gluud LL. Effects of glucagonlike peptide-1 receptor agonists on weight loss: systematic review and metaanalysis of randomized controlled trials. BMJ (2012) 344:d7771. doi:10.1136/ bmj.d7771

21. Astrup A, Rössner S, Van Gaal L, Rissanen A, Niskanen L, AI Hakim $\mathrm{M}$, et al. Effects of liraglutide in the treatment of obesity: a randomized, double-blind, placebo-controlled study. Lancet (2009) 374(9701):1600-16. doi:10.1016/S0140-6736(09)61375-1

22. Mummadi RR, Kasturi KS, Chennareddygari S, Sood GK. Effects of bariatric surgery on nonalcoholic fatty liver disease: systematic review and metaanalysis. Clin Gastroenterol Hepatol (2008) 6(12):1396-402. doi:10.1016/j.cgh. 2008.08.012

23. Chavez-Tapia NC, Tellez-Avila FI, Barrientos-Gutierrez T, Mendez-Sanchez N, Lizardi-Cervera J, Uribe M. Bariatric surgery for non-alcoholic steatohepatitis in obese patients. Cochrane Detabase Syst Rev (2010) 20(1):CD007340. doi:10.1002/14651858.CD007340.pub2

24. Knop FK. Resolution of type 2 diabetes following gastric bypass surgery: involvement of gut-derived glucagon and glucagonotropic signalling? Diabetologia (2009) 52(11):2270-6. doi:10.1007/s00125-009-1511-8

25. Sasaki A, Wakabayashi G, Yonei Y. Current status of bariatric surgery in Japan and effectiveness in obesity and diabetes. J Gastroenterol (2014) 49(1):57-63. doi:10.1007/s00535-013-0802-5

26. Mells JE, Anania FA. The role of gastrointestinal hormones in hepatic lipid metabolism. Semin Liver Dis (2013) 33(4):343-57. doi:10.1055/s-0033-1358527

27. Falken Y, Hellstrom PM, Holst JJ, Naslund E. Changes in glucose homeostasis after Roux-en-Y gastric bypass surgery for obesity at day three, two months, and one year after surgery: role of gut peptides. J Clin Endocrinol Metab (2011) 96(7):2227-35. doi:10.1210/jc.2010-2876

28. Barker KB, Palekar NA, Bowers SP, Goldberg JE, Pulcini JP, Harrison SA. Nonalcoholic steatohepatitis: effect of Roux-en-Y gastric bypass surgery. Am J Gastroenterol (2006) 101(2):368-73. doi:10.1111/j.1572-0241.2006.00419.x

29. Tiikkainen M, Bergholm R, Vehkavaara S, Rissanen A, Häkkinen AM, Tamminen $\mathrm{M}$, et al. Effects of identical weight loss on body composition and features of insulin resistance in obese women with high and low liver fat content. Diabetes (2003) 52(3):701-7. doi:10.2337/diabetes.52.3.701

30. Silverman EM, Sapala JA, Appelman HD. Regression of hepatic steatosis in morbidly obese persons after gastric bypass. Am J Clin Pathol (1995) 104(1): 23-31.

31. Mattar SG, Velcu LM, Rabinovitz M, Demetris AJ, Krasinskas AM, BarinasMitchell E, et al. Surgically-induced weight loss significantly improves nonalcoholic fatty liver disease and the metabolic syndrome. Ann Surg (2005) 242(4):610-620. doi:10.1097/01.sla.0000179652.07502.3f

32. Clark JM, Alkhuraishi AR, Solga SF, Alli P, Diehl AM, Magnuson TH. Roux-en-Y gastric bypass improves liver histology in patients with non-alcoholic fatty liver disease. Obes Surg (2005) 13(7):1180-6.

33. Mottin CC, Moretto M, Padoin AV, Kupski C, Swarowsky AM, Glock L, et al. Histological behavior of hepatic steatosis in morbidly obese patients after weight loss induced by bariatric surgery. Obes Surg (2005) 15(6):788-93. doi:10.1381/0960892054222830

34. Klein S, Mittendorfer B, Eagon JC, Patterson B, Grant L, Feirt N, et al. Gastric bypass surgery improves metabolic and hepatic abnormalities associated with nonalcoholic fatty liver disease. Gastroenterology (2006) 130(6):1564-72. doi:10.1053/j.gastro.2006.01.042

35. Csendes A, Smok G, Burgos AM. Histological findings in the liver before and after gastric bypass. Obes Surg (2006) 16(5):607-11. doi:10.1381/ 096089206776944904

36. de Almeida SR, Rocha PR, Sanches MD, Leite VH, da Silva RA, Diniz MT, et al. Roux-en-Y gastric bypass improves the nonalcoholic steatohepatitis (NASH) of morbid obesity. Obes Surg (2006) 16(3):270-8. doi:10.1381/ 096089206776116462

37. Furuya CK Jr, de Oliveira CP, de Mello ES, Faintuch J, Raskovski A, Matsuda $\mathrm{M}$, et al. Effects of bariatric surgery on nonalcoholic fatty liver disease: preliminary findings after 2 years. J Gastroenterol Hepatol (2007) 22(4):510-4. doi:10.1111/j.1440-1746.2007.04833.x

38. Liu X, Lazenby AJ, Clements RH, Jhala N, Abrams GA. Resolution of nonalcoholic steatohepatitis after gastric bypass surgery. Obes Surg (2007) 17(4):486-92. doi:10.1007/s11695-007-9086-2

39. Weiner RA. Surgical treatment of non-alcoholic steatohepatitis and nonalcoholic fatty liver disease. Dig Dis (2010) 28(1):274-9. doi:10.1159/000282102

40. Meretto M, Kupski C, da Silva VD, Padoin AV, Mottin CC. Effect of bariatric surgery on liver fibrosis. Obes Surg (2012) 22(7):1044-9. doi:10.1007/s11695011-0559-y

41. Vargas V, Allende H, Lecube A, Salcedo MT, Baena-Fustegueras JA, Frot JM, et al. Surgically induced weight loss by gastric bypass improves non alcoholic 
fatty liver disease in morbid obese patients. World J Hepatol (2012) 4(12):382-8. doi:10.4254/wjh.v4.i12.382

42. Tai CM, Huang CK, Hwang JC, Chiang H, Chang CY, Lee CT, et al. Improvement of nonalcoholic fatty liver disease after bariatric surgery in morbidly obese Chinese patients. Obes Surg (2012) 22(7):1016-21. doi:10.1007/s11695-011-0579-7

43. Himpens J, Dapri G, Cadière GB. A prospective randomized study between laparoscopic gastric banding and laparoscopic isolated sleeve gastrectomy: results after 1 and 3 years. Obes Surg (2006) 16:1450-6. doi:10.1381/ 096089206778869933

44. Hutter MM, Schirmer BD, Jones DB, Ko CY, Cohen ME, Merkow RP, et al. First report from the American College of Surgeons Bariatric Surgery Center Network: laparoscopic sleeve gastrectomy has morbidity and effectiveness positioned between the band and the bypass. Ann Surg (2011) 254(3):410-20. doi:10.1097/SLA.0b013e31822c9dac

45. Buckwald H, Oien DM. Metabolic/bariatric surgery worldwide 2011. Obes Surg (2013) 23(4):427-36. doi:10.1007/s11695-012-0864-0

46. Dixon JB, Bhathal PS, Hughes NR, Obrien PE. Nonalcoholic fatty liver disease: improvement in liver histological analysis with weight loss. Hepatology (2004) 39(6):1647-54. doi:10.1002/hep.20251

47. Dixon JB, Bhathal PS, Obrien PE. Weight loss nad non-alcoholic fatty liver disease: falls in gamma-glutamyl transferase concentrations are associated with histologic improvement. Obes Surg (2006) 16(10):1278-86. doi:10.1381/ 096089206778663805

48. Mathurin P, Hollebecque A, Arnalsteen L, Buob D, Leteurtre E, Caiazzo R, et al. Prospective study of the long-term effects of bariatric surgery on liver injury in patients without advanced disease. Gastroenterology (2009) 137(2):532-40. doi:10.1053/j.gastro.2009.04.052

49. Karcz WK, Krawczykowski D, Kuesters S, Marjanovic G, Kulemann B, Grobe H, et al. Influence of sleeve gastrectomy on NASH and type 2 diabetes mellitus. J Obes (2011) 2011:765473. doi:10.1155/2011/765473

50. Umemura A, Sasaki A, Nitta H, Otsuka K, Suto T, Wakabayashi G. Effects of changes in adipocyte hormones and visceral adipose tissue and the reduction of obesity-related comorbidities after laparoscopic sleeve gastrectomy in Japanese patients with severe obesity. Endocr J (2014) 61(4):381-91. doi:10.1507/endocrj. EJ13-0524

51. Keshishian A, Zahriya K, Willes EB. Duodenal switch has no detrimental effects on hepatic function and improves hepatic steatohepatitis after 6 months. Obes Surg (2005) 15(10):1418-23. doi:10.1381/096089205774859290

52. Kral JG, Thung SN, Brion S, Hould FS, Lebel S, Marceau S, et al. Effects of surgical treatment of the metabolic syndrome on liver fibrosis and cirrhosis. Surgery (2004) 135(1):48-58. doi:10.1016/j.surg.2003.10.003

53. Browning JD, Horton JD. Molecular mediators of hepatic steatosis and liver injury. J Clin Invest (2004) 114(2):147-52. doi:10.1172/JCI200422422
54. Sung KC, Kim SH. Interrelationship between fatty liver and insulin resistance in the development of type 2 diabetes. J Clin Endocrinol Metab (2011) 96(4):1093-7. doi:10.1210/jc.2010-2190

55. Ionut V, Burch M, Youdim A, Bergman RN. Gastrointestinal hormones and bariatric surgery-induced weight loss. Obesity (2013) 21(6):1093-103. doi:10. 1002/oby.20364

56. Patti ME, Houten SM, Bianco AC, Bernier R, Larsen PR, Holst JJ, et al. Serum bile acids are higher in humans with prior gastric bypass: potential contribution to improved glucose and lipid metabolism. Obesity (2009) 17(9):1671-7. doi:10.1038/oby.2009.102

57. Haluzíková D, Lacinová Z, Kaválková $\mathrm{P}$, Drápalová J, Kř́ǐzová J, Bártlová M, et al. Laparoscopic sleeve gastrectomy differentially affects serum concentrations of FGF-19 and FGF-21 in morbidly obese subjects. Obesity (Silver Spring) (2013) 21(7):1335-42. doi:10.1002/oby.20208

58. Pournaras DJ, Glicksman C, Vincent RP, Kuganolipava S, Alaghband-Zadeh J, Mahon D, et al. The role of bile after Roux-en-Y gastric bypass in promoting weight loss and improving glycaemic control. Endocrinology (2012) 153(8):3613-9. doi:10.1210/en.2011-2145

59. Vest AR, Heneghan HM, Agarwal S, Schauer PR, Young JB. Bariatric surgery and cardiovascular outcomes: a systematic review. Heart (2012) 98(24):1763-77. doi:10.1136/heartjnl-2012-301778

60. Poirier P, Auclair A. Role of bariatric surgery in diabetes. Curr Cardiol Rep (2014) 16(2):444. doi:10.1007/s11886-013-0444-5

61. Laferrere B. Diabetes remission after bariatric surgery: is it just the incretins? Int J Obes (Lond) (2011) 35(Suppl 3):S22-5. doi:10.1038/ijo.2011.143

Conflict of Interest Statement: The authors declare that the research was conducted in the absence of any commercial or financial relationships that could be construed as a potential conflict of interest.

Received: 19 May 2014; accepted: 25 September 2014; published online: 27 October 2014

Citation: Sasaki A, Nitta H, Otsuka K, Umemura A, Baba S, Obuchi T and Wakabayashi $G$ (2014) Bariatric surgery and non-alcoholic fatty liver disease: current and potential future treatments. Front. Endocrinol. 5:164. doi: 10.3389/fendo.2014.00164

This article was submitted to Neuroendocrine Science, a section of the journal Frontiers in Endocrinology.

Copyright (c) 2014 Sasaki, Nitta, Otsuka, Umemura, Baba, Obuchi and Wakabayashi. This is an open-access article distributed under the terms of the Creative Commons Attribution License (CC BY). The use, distribution or reproduction in other forums is permitted, provided the original author(s) or licensor are credited and that the original publication in this journal is cited, in accordance with accepted academic practice. No use, distribution or reproduction is permitted which does not comply with these terms. 